\title{
Flexor Carpi Radialis Brevis Resection for Treatment of a Distal Radius Fracture: A Case Report
}

\author{
Ryan L. Werntz, DO ${ }^{1}$ Andrew J. Hadeed, DO ${ }^{1}$ Giacomo L. Cappelleti, MD ${ }^{2}$ Jorge L. Orbay, MD² \\ 1 Orthopaedic Surgery Residency Program, Larkin University Hospital, \\ Miami, Florida \\ 2 Department of Orthopaedic Surgery, The Miami Hand \& Upper \\ Extremity Institute, Miami, Florida \\ Address for correspondence Jorge L. Orbay, MD, The Miami Hand \& \\ Upper Extremity Institute, 8905 SW 87th Avenue, Suite 201, Miami, \\ FL 33176 (e-mail: jlorbay@gmail.com).
}

J Wrist Surg 2021;10:536-538.

\begin{abstract}
Keywords

- anomalous muscle

- distal radius fracture

- flexor carpi radialis brevis

- resection

The flexor carpi radialis brevis (FCRB) is an anomalous muscle of the forearm that is only present in 3.5 to $8.6 \%$ of the population. In the case of volar plating of distal radius fractures, the FCRB inhibits proper fracture exposure and thus hinders proper reduction. A 78-year-old female presented with right distal radius fracture which necessitated internal fixation. Following mobilization and retraction of the flexor carpi radialis tendon, an anomalous muscle belly was identified as the FCRB. With continued difficulty in exposure and fracture site reduction, resection of the FCRB was performed. The patient was able to return to her activities of daily living without pain and demonstrated no appreciable functional deficit. This case report demonstrates a distal radius fracture where FCRB resection was used, resulting in no detrimental clinical outcomes.
\end{abstract}

The flexor carpi radialis brevis (FCRB) is a well-known anomalous muscle of the forearm that is only present in 3.5 to $8.6 \%$ of the population. ${ }^{1,2}$ First described by Fano in 1851 , knowledge of its existence was expanded upon by Woods in 1867 calling the muscle a "short radiocarpal flexor" and gave the name flexor carpi radialis brevis vel profundus. $^{3-5}$ Innervated by the anterior interosseous nerve, the FCRB typically originates from the distal aspect of the volarradial surface of the radius with extremely variable insertion points. ${ }^{3,4}$ These insertion points include, but are not limited to, the second, third, or fourth metacarpals as well as the trapezium or the capitate. ${ }^{3}$

Along with its variable insertion points, the FCRB has also been known to bring with it additional anatomical variations, namely a hypoplastic pronator quadratus. ${ }^{3,6}$ Not only this, but the pronator quadratus has also been reported to be completely absent in some patients with an FCRB. ${ }^{7}$ Dodds et al in his cadaveric study showed that the FCRB muscle lays across the radial aspect of the radius and is thought to compress the pronator quadratus leading to its inadequate development. ${ }^{3,8}$

In the past, the FCRB was most commonly seen through cadaveric assessment. ${ }^{5,8}$ However, as the incidence of surgical treatment of distal radius fractures increases, encounters with this anomalous muscle are increasing in parallel. ${ }^{1,4}$ As the encounters increase, it is of paramount importance that surgeons are aware of their different options in dealing with anatomical variants, and the effects they can have on their patient population. We aim to describe one such experience and how our treatment plan lead to a satisfactory outcome in one specific patient. received

November 10, 2020

accepted

February 8, 2021

published online

April 14, 2021
DOI https://doi.org/ $10.1055 / \mathrm{s}-0041-1726407$ ISSN 2163-3916.

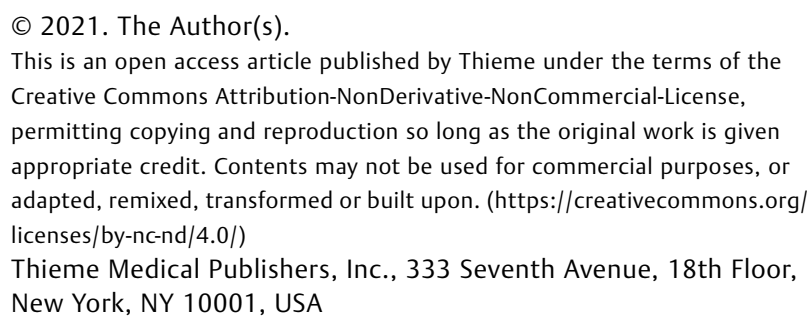




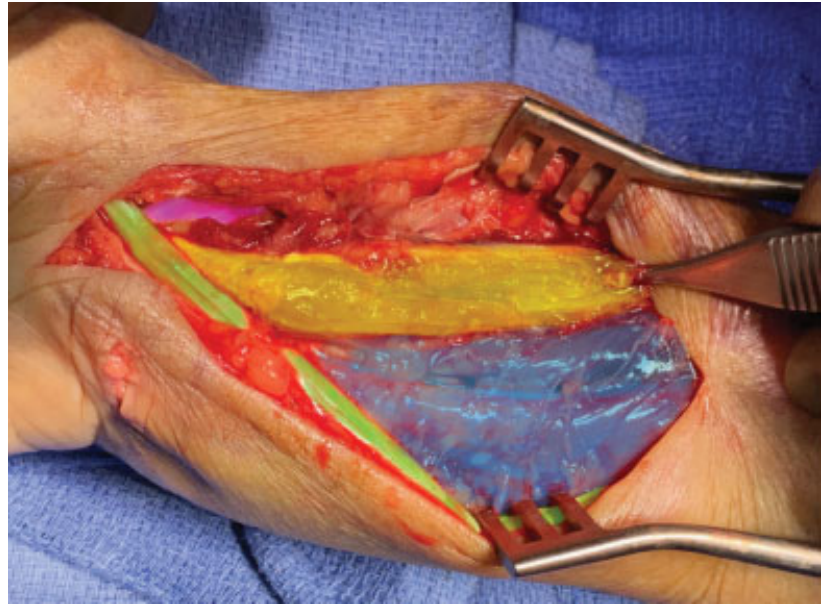

Fig. 1 Volar exposure with anomalous anatomy of flexor carpi radialis brevis muscle (yellow), flexor carpi radialis tendon (green), flexor pollicis longus muscle (blue), and brachioradialis tendon (pink).

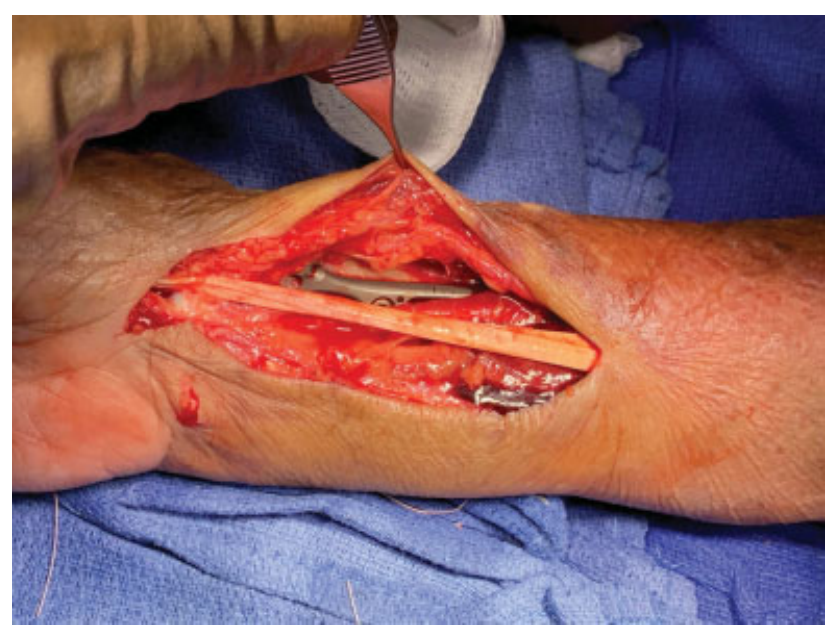

Fig. 3 After flexor carpi radialis brevis excision and completion of fracture fixation.

\section{Case Report}

An active 78-year-old female presented to our clinic complaining of right wrist pain and deformity 3 days after a fall onto an out-stretched hand. Radiographs demonstrated a right distal radius fracture with intra-articular fracture displacement and significant dorsal angulation. After discussing with patient and family the various treatment options, open reduction and internal fixation was chosen to be the treatment most beneficial for our patient.

On the day of surgery, a standard extended flexor carpi radialis (FCR) approach was performed. A 9-cm incision was made along the FCR with a zig-zag across the wrist flexion creases. $^{9}$ After the FCR tendon was mobilized, the deep portion of the FCR sheath was incised and the FCR retracted medially. At this time, it was made apparent that an anomalous muscle belly was abutting the flexor pollicis longus radially and was determined to be an FCRB (-Fig. 1). The muscle was found to originate over the distal one-third of the

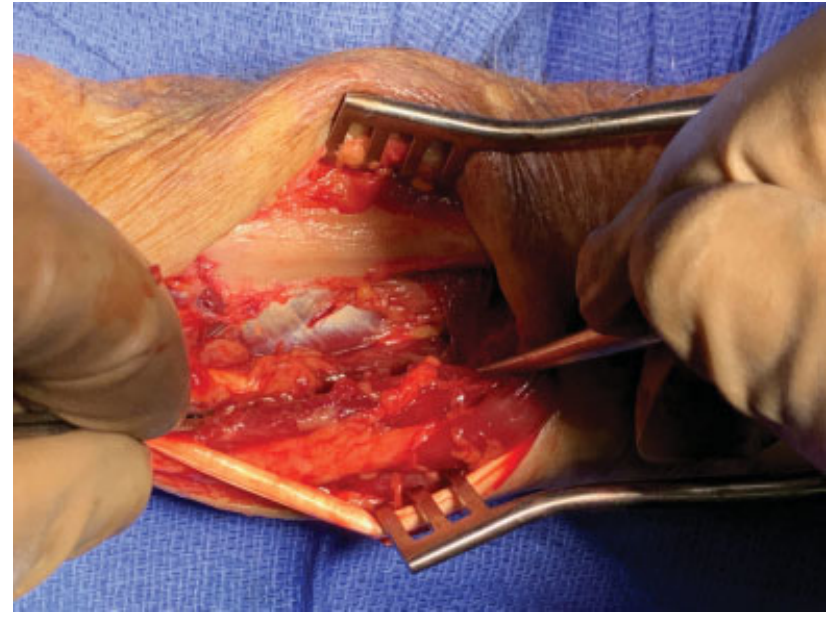

Fig. 2 After extensor carpi radialis brevis excision, an associated hypoplastic pronator quadratus from the presence of flexor carpi radialis brevis muscle is seen.

radius and its tendon coursed parallel to the FCR tendon. We then mobilized the FCRB muscle and gained access to the distal aspect of the radius and the radial septum. ${ }^{10}$ Dissection continued to be performed into the space of Parona where the pronator quadratus was found to be significantly decreased in width, consisting of only approximately $50 \%$ of a typical radial insertion footprint (-Fig. 2). This pronator quadratus was found to have a typical ulnar origin, but only inserted on the ulnar half of the volar distal radius surface showing consistent findings with Dodd's study in $2006 .^{8}$

Continuing on with our dissection, we found it extremely difficult to expose and reduce the fracture site as the ECRB could not be retracted ulnarly due to the inability to mobilize its distal tendon without transection. Also, the vascular supply to the FCRB was seen to arise from the radial artery and ulnar mobilization would have resulted in its devascularization. Therefore, excision of the FCRB was performed. The origin was elevated with a key elevator and the tendon was transected distally. Once this was complete, the remainder of the procedure was routinary and performed with ease. Closure was performed in the normal fashion ( - Fig. 3 ).

Patient was seen at 1 and 5 weeks postoperatively, demonstrating an expected course of healing with no significant detriment during rehabilitation. At 11 weeks, the patient showed full range of motion with fully regained wrist strength compared with the contralateral side. She was able to return to her activities of daily living without pain or limitation. There was no appreciable functional deficit from the absence of the FCRB.

\section{Discussion}

Surgeons should be well versed in anomalous anatomy that they may come into contact with. This knowledge facilitates making alternate decisions during dissection and exposure, decreasing operative time and risk of complications.

In his study, Lee et al described two separate cases of an FCRB encountered during operative fracture care, one in a 
patient with both bone forearm fracture, and one in a distal radius fracture; both treated with ORIF. ${ }^{8}$ In both of his cases, it was elected to retain the FCRB due to additional procedures not being included during preoperative discussion. While retention of an FCRB may be an option in both bone fractures, in our experience doing so in distal radius fractures significantly reduces anatomic exposure and thus proper fracture reduction. Moreover, it has been shown that FCRBs can be the source of clinical problems, adding to the argument for removal. ${ }^{1}$

The aim of this case report is to show that in some cases, the proper treatment of a distal radius fracture may necessitate resection of an anomalous FCRB and that this will not cause a detriment in the patient's clinical outcome.

Funding

None.

\section{Conflict of Interest}

None declared.

\section{Acknowledgments}

The authors would like to thank Lauren Vernon PhD for her help with the editorial review and submission process.

\section{References}

1 Mantovani G, Lino W Jr, Fukushima WY, Cho AB, Aita MA. Anomalous presentation of flexor carpi radialis brevis: a report of six cases. J Hand Surg Eur Vol 2010;35(03):234-235

2 Kosiyatrakul A, Luenam S, Prachaporn S. Symptomatic flexor carpi radialis brevis: case report. J Hand Surg Am 2010;35(04):633-635

3 Andring N, Kennedy SA, Iannuzzi NP. Anomalous forearm muscles and their clinical relevance. J Hand Surg Am 2018;43(05):455-463

4 Lee Y-M, Song S-W, Sur Y-J, Ahn CY. Flexor carpi radialis brevis: an unusual anomalous muscle of the wrist. Clin Orthop Surg 2014;6 (03):361-364

5 Carleton A. Flexor carpi radialis brevis vel profundus. J Anat 1935; 69(Pt 2):292-293

6 Kang L, Carter T, Wolfe SW. The flexor carpi radialis brevis muscle: an anomalous flexor of the wrist and hand. A case report. J Hand Surg Am 2006;31(09):1511-1513

7 Tountas CP, Bergman RA. Anatomic Variations of the Upper Extremity. New York, NY: Churchill Livingstone; 1993:139-140

8 Dodds SD. A flexor carpi radialis brevis muscle with an anomalous origin on the distal radius. J Hand Surg Am 2006;31(09):1507-1510

9 Orbay JL, Badia A, Indriago IR, et al. The extended flexor carpi radialis approach: a new perspective for the distal radius fracture. Tech Hand Up Extrem Surg 2001;5(04):204-211

10 Orbay JL, Gray R, Vernon LL, Sandilands SM, Martin AR, Vignolo $\mathrm{SM}$. The EFCR approach and the radial septum-understanding the anatomy and improving volar exposure for distal radius fractures: imagine what you could do with an extra inch. Tech Hand Up Extrem Surg 2016;20(04):155-160 